\title{
Hopping mobility of charge carriers in disordered organic host- guest systems: Dependence on the charge-carrier concentration
}

\author{
Citation for published version (APA): \\ Coehoorn, R. (2007). Hopping mobility of charge carriers in disordered organic host-guest systems: \\ Dependence on the charge-carrier concentration. Physical Review B, 75(15), 155203-1/10. [155203]. \\ https://doi.org/10.1103/PhysRevB.75.155203
}

DOI:

10.1103/PhysRevB.75.155203

Document status and date:

Published: 01/01/2007

\section{Document Version:}

Publisher's PDF, also known as Version of Record (includes final page, issue and volume numbers)

\section{Please check the document version of this publication:}

- A submitted manuscript is the version of the article upon submission and before peer-review. There can be important differences between the submitted version and the official published version of record. People interested in the research are advised to contact the author for the final version of the publication, or visit the DOI to the publisher's website.

- The final author version and the galley proof are versions of the publication after peer review.

- The final published version features the final layout of the paper including the volume, issue and page numbers.

Link to publication

\footnotetext{
General rights

- You may freely distribute the URL identifying the publication in the public portal. follow below link for the End User Agreement:

www.tue.nl/taverne

\section{Take down policy}

If you believe that this document breaches copyright please contact us at:

openaccess@tue.nl

providing details and we will investigate your claim.
}

Copyright and moral rights for the publications made accessible in the public portal are retained by the authors and/or other copyright owners and it is a condition of accessing publications that users recognise and abide by the legal requirements associated with these rights.

- Users may download and print one copy of any publication from the public portal for the purpose of private study or research.

- You may not further distribute the material or use it for any profit-making activity or commercial gain

If the publication is distributed under the terms of Article $25 \mathrm{fa}$ of the Dutch Copyright Act, indicated by the "Taverne" license above, please 


\title{
Hopping mobility of charge carriers in disordered organic host-guest systems: Dependence on the charge-carrier concentration
}

\author{
R. Coehoorn \\ Philips Research Laboratories, High Tech Campus 4, 5656 AE Eindhoven, The Netherlands, \\ and Department of Applied Physics, Eindhoven University of Technology, P.O. Box 513, 5600 MB, The Netherlands
}

(Received 25 October 2006; published 12 April 2007)

\begin{abstract}
The hopping mobility of charge carriers in disordered organic host-guest systems with a bimodal Gaussian density of states is studied. Two semianalytical models are used, viz. a relatively simple Mott-type model and a more advanced but computationally less efficient effective medium model. The latter model has been generalized, in order to be able to include the effect of different wave function extensions of the host and guest molecules. It is shown that energetic disorder can result in a pronounced charge carrier concentration dependence of the mobility. This provides an explanation so far for unresolved issues concerning the guest concentration dependence of the measured hole mobility in some well-characterized host-guest systems. It is also argued that treating the mobility as a carrier concentration dependent quantity is highly relevant to the modeling of carrier transport in organic light emitting devices (OLEDs), consisting of an organic matrix material with embedded fluorescent or phosphorescent dye molecules.
\end{abstract}

DOI: 10.1103/PhysRevB.75.155203

\section{INTRODUCTION}

Disordered organic semiconducting host-guest systems are employed in several types of devices. In organic light emitting diodes (OLEDs), e.g., hole transporting guest molecules have been used to decrease the barrier for hole injection, ${ }^{1,2}$ fluorescent dye molecules can be added to tune the emission color $^{3}$ and phosphorescent dye molecules containing heavy metal atoms are used to harvest both singlet and triplet excitons. ${ }^{4}$ Fluorescent dye molecules have also been used to obtain light amplification in organic thin films using cascade energy transfer. ${ }^{5}$ In xerography, molecularly doped polymers are used as photoreceptors. ${ }^{6}$ In any organic electronic material, guest molecules can also be present as an unintended result of nonideal preparation processes. The presence of guest molecules has a strong effect on the transport properties if their HOMO (highest occupied molecular orbital) states and/or LUMO (lowest unoccupied molecular orbital) states lie within the energy gap of the host material. At small concentrations, such guest molecules give rise to a decrease of the mobility, $\mu$, as a result of charge carrier trapping on the molecule. In some systems, this only continues until, at certain guest concentration, a minimum in the mobility is reached. For larger guest concentrations, the mobility increases with concentration as a result of direct hopping between the guest molecules. ${ }^{7}$

The effect of guest molecules on the mobility has been modeled in various ways. When the energy levels of the guest molecules are well below the host energy levels in between which hopping takes place, the guest sites can be viewed as traps. Within the so-called multiple-trap-andrelease model, the effective mobility is given by $\mu(x)$ $=f(x) \mu(0)$, where $x$ is the guest molecule concentration, $\mu(0)$ is the mobility in the absence of guest molecules, and $f(x)$ is the fraction of charge carriers that on average reside on a host site. Within this model, direct guest-to-guest hopping is neglected, which is appropriate at sufficiently small guest concentrations. Neglecting disorder of the host and guest energy levels, and assuming sufficiently deep trap energies, so
PACS number(s): 72.20.Ee, 72.80.Le, 72.80.Ng

that Boltzmann statistics is applicable, the mobility reduction is then given by the Hoesterey-Letson (HL) formula

$$
\frac{\mu(x)}{\mu(0)}=\frac{1}{1+x \exp \left(\frac{\Delta}{k_{B} T}\right)},
$$

where $\Delta$ is the energy difference between the host and trap energy levels, and $k_{\mathrm{B}} T$ is the thermal energy. ${ }^{8}$ The presence of guest molecules thus reduces the mobility by a factor 2 for a concentration $x_{1 / 2}=\exp \left(-\Delta /\left(k_{\mathrm{B}} T\right)\right)$. This defines the boundary between the host-to-host (HH) hopping regime $\left(x<x_{1 / 2}\right)$, in which the presence of the guest molecules does not significantly affect the mobility, and the host-guest-host (HGH) hopping regime $\left(x_{1 / 2}<x<x_{\min }\right)$. In the latter regime, trapping at guest molecules reduces the mobility significantly. A second crossover, to a guest-to-guest (GG) hopping regime, occurs at the guest concentration $x_{\min }$ at which the mobility is minimal, if there is such a minimum. Qualitatively, the results of time-of-flight measurements of the mobility in organic host-guest systems provide support for this picture. ${ }^{9}$ However, proper quantitative analyses should take the disorder of the energies of the host and guest sites into account. This was done by Wolf et al., ${ }^{10,11}$ Fishchuk et al. ${ }^{12}$ and Arkhipov, ${ }^{13}$ who developed quantitative models for the mobility in host-guest systems, in all cases assuming a bimodal Gaussian density of states (DOS). Recently, Fishchuk et al. extended their model in order to include the transition around $x=x_{\min }$ between the HGH and GG hopping regimes. ${ }^{14}$

In spite of this progress, the theoretical models developed so far still lack an important ingredient: the mobility does not only depend on the guest concentration, but also on the charge carrier concentration, $c$. It is the purpose of this paper to demonstrate the relevance of this effect, by showing the results from a model for the dependence of the mobility on $x$ and $c$, for the case of a bimodal Gaussian DOS. We discuss the relevance of this effect for OLEDs, and argue that it might provide an explanation for an unresolved discrepancy between the $\mu(x)$ curves observed from time-of-flight (TOF) 
experiments for certain well-characterized amine-based hostguest systems and the so far available models. ${ }^{15,16}$ The observed values of $x_{1 / 2}$ and the rate of decrease of the mobility beyond $x_{1 / 2}$ were for some systems significantly larger than predicted from the results of Monte Carlo calculations. ${ }^{10}$ The mobilities were derived from the trajectories of single particles in an otherwise empty box, so that a possible charge carrier concentration dependence of the mobility was neglected.

The notion that the hopping mobility in disordered organic systems can depend on the carrier concentration is not new. ${ }^{17,18}$ For a unimodal Gaussian DOS, several semianalytical models lead consistently to such a dependence above a certain minimum critical carrier density (Ref. 19 and references therein). Numerical results from a Master Equation approach and an analysis of the experimental current-voltage characteristics of single-carrier polymer LED type are fully consistent with the semianalytical results. ${ }^{20}$ In a unimodal Gaussian DOS the mobility becomes carrier concentration dependent when the Fermi energy (with respect to the top of the Gaussian) is equal to $E_{\mathrm{F}}=-\sigma_{\mathrm{DOS}} 2 /\left(k_{\mathrm{B}} T\right)$, with $\sigma_{\mathrm{DOS}}$ the width of the Gaussian DOS, i.e., at a crossover carrier concentration $^{19}$

$$
c_{\text {cross-over }}=\frac{1}{2} \exp \left[-\frac{1}{2} \hat{s}^{2}\right],
$$

with $\hat{s} \equiv \sigma_{\text {DOS }} /\left(k_{\mathrm{B}} T\right)$. Below this carrier concentration, the carriers act as independent particles (Boltzmann limit): the shape of the density of occupied states is independent of the carrier concentration. In contrast, above $c_{\text {crossover }}$ the occupation probability of the deepest guest states is so large that their effectiveness as trap sites decreases significantly with increasing carrier concentration. As a result, the mobility increases with increasing carrier concentration. In disordered organic systems, $\sigma$ varies typically from 0.05 to $0.15 \mathrm{eV}$, so that at room temperature $\hat{s}$ varies typically from 2 to 6 . For $\hat{s}=5$, e.g., the crossover concentration is approximately $10^{-6}$. The actual carrier concentration in $80 \mathrm{~nm}$ single-layer OLEDs, at typical operational conditions, is significantly larger, viz. typically $10^{-5}$ to $10^{-4}$ in the center of the layer, and even larger near the injecting electrodes. In multilayer OLEDs, containing electron and hole blocking layers, the carrier concentration in the emissive layers can be of the order $10^{-3} \cdot{ }^{21}$ Disorder is thus expected to give rise to an appreciable carrier concentration dependence of the mobility in such systems. The effect is expected to be even larger for host-guest systems, where the relevant carrier concentration is the ratio between the carrier density and the (small) guest density.

This paper is organized as follows. The theoretical models used are discussed in Sec. II. We have developed a model for treating the mobility in the $\mathrm{HGH}-\mathrm{GG}$ transition region that goes beyond the approach presented by Fishchuk et al. ${ }^{14}$ in two respects: (i) the carrier concentration dependence of the effect is taken into account, and (ii) the possibility that the wave function extensions of the host and guest molecules are different is included. In Sec. III, results are given for a bimodal Gaussian DOS with various widths of the host and guest DOS, and in Sec. IV, a quantitative analysis of these results is given. In Sec. V the model is used to analyse TOF mobilities of amine-based systems, as discussed above. Section VI contains a summary and concluding remarks.

\section{THEORETICAL MODELS}

In a previous publication, on the mobility in a unimodal Gaussian DOS, we have presented a critical comparison of the carrier concentration dependence of the mobility, as deduced from all available semianalytical models. ${ }^{19}$ Perhaps the simplest, and yet successful, model is the Mott-type model developed by Martens et al. 19,22 ("Martens model"). We have found that its relative simplicity makes it possible to calculate the carrier and guest concentration dependent mobility for transport in a bimodal Gaussian DOS in a computationally efficient way. From a physical and computational point of view, the model developed by Movaghar and Shirmacher (MS), using a modified effective medium approximation, ${ }^{19,23}$ represents the other extreme. The MS model is physically more rigorous than the Martens model. However, for the case of the mobility in a unimodal Gaussian DOS, a comparison with the numerically exact results from a Master Equation approach did not allow us to conclude that the MS model is indeed significantly better than the more simple and computationally much more efficient Martens model. ${ }^{19}$ In Sec. IV, we show that for a bimodal DOS the MS model is in some cases better than the Martens model, although the latter model already contains all the essential physics. First, we find that the MS model provides a better description of the mobility at the transition between the two $\mathrm{HGH}$ and GG hopping regimes. Second, the use of the MS model makes it possible to consider host and guest molecules with different extensions of their wave functions. In this section, we briefly introduce both methods. Only the mobility in the limit of zero applied field is considered.

\section{A. Mott-type model Martens et al.}

The total DOS is assumed to be the sum of a Gaussian host and guest DOS, centered at energies $E=0$ and $E=-\Delta$, with widths $\sigma_{\mathrm{DOS}, \mathrm{h}}$ and $\sigma_{\mathrm{DOS}, \mathrm{g}}$, and with volume site densities $N_{\mathrm{h}}$ and $N_{\mathrm{g}}$, respectively. The site positions are random. The guest concentration is defined as $x \equiv N_{\mathrm{g}} / N_{\mathrm{t}}$, where $N_{\mathrm{t}}$ is the total site density, $N_{\mathrm{t}} \equiv N_{\mathrm{h}}+N_{\mathrm{g}}$. Millar-Abrahams hopping rates between localized sites are assumed (see Ref. 19 and references therein). The hopping rate between two sites at a distance $R$ is proportional to $\exp (-2 \alpha R)$, where $\alpha$ is the inverse of the effective extension of the localized wave functions. Unless specified otherwise, we assume that $N_{\mathrm{t}} / \alpha^{3}$ $=10^{-3}$ (so that $\alpha^{-1}=0.1 \times a$, with $a$ the average intersite distance), and that $\alpha$ is equal for host and guest sites. Within the Martens model, the conductivity is given by

$$
\sigma=\sigma_{0} \exp \left[-2 \alpha R^{*}-\frac{E^{*}-E_{F}}{k_{B} T}\right],
$$

where $\sigma_{0}$ is a prefactor that will be treated as a constant. The mobility is defined as $\mu \equiv \sigma /(n q)$, with $n$ the carrier density and $q$ the elementary charge. It is assumed to be governed by 
hops over a distance $R^{*}$, from the Fermi energy $E_{\mathrm{F}}$ to an effective "transport energy" $E^{*} . R^{*}$ and $E^{*}$ are determined from the following two requirements: ${ }^{22}$

(i) Each site has on average $B$ neighbor sites within a radius $R^{*}$ and with an energy in the range $\left[E_{\mathrm{F}} ; E^{*}\right]$, and

(ii) $R^{*}$ and $E^{*}$ are taken such that $\sigma$ is maximized.

$B$ is a "percolation parameter." In the original version of the Martens model $B=1$ was taken. However, we have shown in Ref. 19 that the model needs to be modified, and that $B$ should be taken larger. As in Ref. 19, we take here $B=1.969$. This choice affects the guest concentration dependence of the mobility near the mobility minimum, at $x=x_{\min }$, but not the relative mobility decrease upon the introduction of a small concentration of guest molecules. Straightforward application of these two requirements leads to the transcendental equation

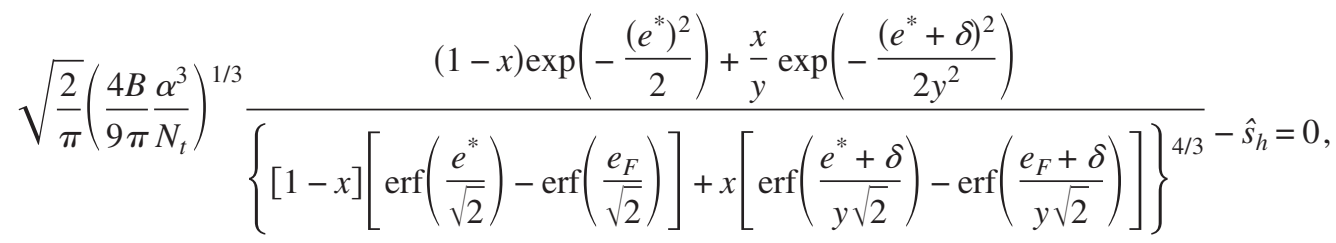

from which $E^{*}$ can be deduced. Here $y=\sigma_{\mathrm{DOS}, \mathrm{g}} / \sigma_{\mathrm{DOS}, \mathrm{h}}, e^{*}=E^{*} / \sigma_{\mathrm{DOS}, \mathrm{h}}$ and $\delta=\Delta / \sigma_{\mathrm{DOS}, \mathrm{h}}, E_{\mathrm{F}} / \sigma_{\mathrm{DOS}, \mathrm{h}}$, and erf is the error function. $R^{*}$ is then given by

$$
R^{*}=\left(\frac{3}{2 \pi} \frac{B}{N_{t}}\right)^{1 / 3} \frac{1}{\left\{[1-x]\left[\operatorname{erf}\left(\frac{e^{*}}{\sqrt{2}}\right)-\operatorname{erf}\left(\frac{e_{F}}{\sqrt{2}}\right)\right]+x\left[\operatorname{erf}\left(\frac{e^{*}+\delta}{y \sqrt{2}}\right)-\operatorname{erf}\left(\frac{e_{F}+\delta}{y \sqrt{2}}\right)\right]\right\}^{1 / 3}} .
$$

The mobility follows by first calculating the Fermi energy, for the temperature and carrier concentration of interest, using Fermi-Dirac statistics, then deriving $E^{*}$ and $R^{*}$ from Eqs. (4) and (5), and finally calculating $\sigma$ from Eq. (3) and $\mu=\sigma /\left(q c N_{\mathrm{t}}\right)$. The hopping process can be described as "variable range hopping" when $R^{*} \gg a$, and as "nearest neighbor hopping" when $R^{*} \approx a$. For typical organic semiconductors with a unimodal Gaussian DOS, with $\sigma$ in the range 0.05 to $0.15 \mathrm{eV}$, the mobility is around room temperature in the nearest neighbor hopping regime.

In this paper we will mostly be interested in the reduction of the mobility due to the presence of guest molecules, i.e., in generalizations of Eq. (1). A key result that has emerged from earlier studies of the mobility in disordered systems with a Gaussian DOS (see Ref. 19 and references therein) is that for small carrier concentrations, in the tail of the DOS, the transport energy level $E^{*}$ and the radius $R^{*}$ are to a very good approximation independent of the carrier concentration. It follows then from Eq. (3) that the conductivity is only dependent on the carrier concentration via $E_{\mathrm{F}}(c)$. The mobility enhancement, defined as the ratio $\mu(c) / \mu(c=0)$, does therefore not depend on the wave function decay length and on the percolation parameter. For the case of a unimodal Gaussian DOS, it was shown in Ref. 19 (Fig. 7) that $\mu(c) / \mu(c=0)$ even does not depend on the precise form of the hopping model that is assumed; an exact expression is given by Eq. (29) in Ref. 19. For a bimodal Gaussian DOS, with guest concentration $x$, it follows using Eq. (3) that the mobility enhancement is given by

$$
\frac{\mu(x, c)}{\mu(0,0)} \equiv \lim _{c_{0} \rightarrow 0} \frac{\sigma(x, c)}{c} \frac{c_{0}}{\sigma\left(0, c_{0}\right)} \cong \lim _{c_{0} \rightarrow 0} \frac{c_{0} \exp \left[\frac{E_{F}(x, c)}{k_{B} T}\right]}{c \exp \left[\frac{E_{F}\left(0, c_{0}\right)}{k_{B} T}\right]} .
$$

This may be viewed as a generalized form of Eq. (1).

For the case of a bimodal Gaussian DOS, Arkhipov has applied the transport energy level concept to obtain a generalized expression for the mobility reduction in the limit of a small carrier concentration. ${ }^{24}$ Using the $E_{\mathrm{F}}(c)$ relationship given by Eq. (A2) in Ref. 19 (an excellent approximation for $\hat{s}>2$ and for carrier concentrations well below the crossover concentration), it follows that $c$ depends in the following way on $E_{\mathrm{F}}$ and $x$ :

$$
c=\exp \left[\frac{E_{F}+\Delta}{k_{B} T}+\frac{1}{2} \hat{s}_{g}^{2}\right] x+\exp \left[\frac{E_{F}}{k_{B} T}+\frac{1}{2} \hat{s}_{h}{ }^{2}\right](1-x) .
$$

For $x \ll 1$, and $\Delta / \sigma_{\mathrm{DOS}, \mathrm{h}} \gg 1$, so that the transport energy level is independent of $x$, the mobility can then be obtained using Eq. (6)

$$
\mu(x, 0) \cong \frac{1}{(1-x)+x \exp \left[\frac{\Delta}{k_{B} T}+\frac{1}{2} \hat{s}_{g}{ }^{2}-\frac{1}{2} \hat{s}_{h}^{2}\right]^{\mu}} \mu(0,0) .
$$

This result agrees for $x \ll 1$ with the slightly more general expression, given without derivation by Arkhipov. ${ }^{24}$ The mo- 
bility has the same functional dependence on $x$ as in the HL formula. However, the guest concentration beyond which the mobility is reduced by a factor of two is increased or decreased by the introduction of disorder, depending on the difference between the Gaussian widths of the host and guest densities of states

$$
x_{1 / 2}=\frac{1}{\exp \left[\frac{\Delta}{k_{B} T}+\frac{1}{2} \hat{s}_{g}{ }^{2}-\frac{1}{2} \hat{s}_{h}{ }^{2}\right]},
$$

for $x_{1 / 2} \ll 1$.

As long as the transport level is independent of $c$ and $x$, Eq. (6) provides an excellent approximation for the carrier concentration dependence of the mobility. The approximation breaks down at large carrier concentrations (above $c \approx 10^{-2}-10^{-1}$ ) and near the guest concentration at which a crossover takes place between the HGH and GG hopping regimes. The wave function decay length, the percolation parameter, and final state filling effects become then important. The results given in Sec. III have been obtained using the full theory [Eqs. (3)-(5)]. However, it is instructive to apply Eq. (6) for the simple case without disorder, i.e., $\sigma_{\mathrm{DOS}, \mathrm{h}}=\sigma_{\mathrm{DOS}, \mathrm{g}}=0$. When the guest and carrier concentrations are small, $E^{*}=0$ and $R^{*}=\left[3 B /\left(4 \pi N_{\mathrm{t}}\right)\right]^{1 / 3}=0.779 a$. The exact expression for the carrier concentration is

$$
c=\frac{x}{1+\exp \left(\frac{-\Delta-E_{F}}{k_{B} T}\right)}+\frac{1-x}{1+\exp \left(-\frac{E_{F}}{k_{B} T}\right)} .
$$

Equation (10) can be written as a quadratic equation in $\exp \left[-E_{\mathrm{F}} /\left(k_{\mathrm{B}} T\right)\right]$, so that using Eq. (6) an analytic expression for $\mu(x, c)$ can be obtained in a straightforward manner. The result is shown graphically in Fig. 1, in the form of a contour plot for the $\log _{10}$ of the mobility reduction, as a function of the carrier and guest concentration, for $x_{1 / 2}=10^{-5}$. At room temperature, this corresponds to $\Delta \approx 0.29 \mathrm{eV}$. In the lower right hand part of the diagram, the HL formula is valid, and in the upper left hand part, the presence of guest molecules does not affect the mobility (small guest concentration and large carrier concentration). In the narrow transition zone

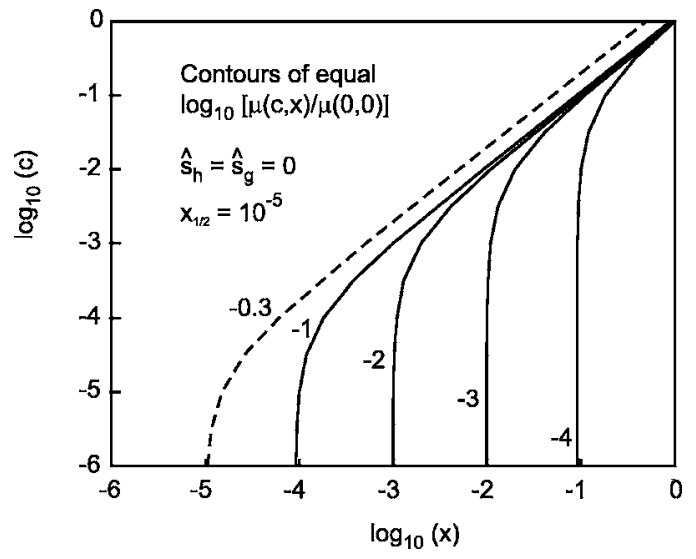

FIG. 1. Guest concentration $(x)$ and carrier concentration $(c)$ dependence of the mobility in a host-guest system without energetic disorder $\left(\hat{s}_{\mathrm{h}}=\hat{s}_{\mathrm{g}}=0\right)$ and with the energy difference between the host and guest states, $\Delta=11.51 k_{\mathrm{B}} T$, such that $x_{1 / 2}=\exp \left[-\Delta /\left(k_{\mathrm{B}} T\right)\right]$ $=10^{-5}$. The figure shows contours of equal mobility, expressed as $\log _{10}[\mu(c, x) / \mu(0,0)]$.

near the line $c=x$, for $x>x_{1 / 2}$, the mobility is strongly dependent on the carrier concentration.

\section{B. Movaghar-Shirmacher (MS) model}

Within the effective medium approximation used by Movaghar and Schirmacher, the conductivity is expressed as an integral over contributions from all sites. ${ }^{23}$ A slightly simplified expression for the conductivity, introduced in Ref. 19, is

$$
\sigma \cong \frac{q^{2}}{k_{B} T} \frac{\left\langle R^{2}\right\rangle}{6} B_{M S} \nu_{0} \int_{-\infty}^{+\infty} g(E) \sigma_{1}(E) d E
$$

where $\left\langle R^{2}\right\rangle=A N_{\mathrm{t}}^{-2 / 3}$ (with $A$ a number of the order 1) is the average of the square of the hopping distance, $B_{\mathrm{MS}}$ is a "percolation correction factor" with a numerical value $B_{\mathrm{MS}}$ $=\exp (1), g(E)$ is the density of states, and $\nu_{0}$ is the hopping attempt frequency. Throughout the paper, we assume that $\nu_{0}$ does not depend on the molecules in between which the hopping takes place. The dimensionless function $\sigma_{1}(E)$, which gives the contribution from sites with energy $E$, is given by

$$
\sigma_{1}(E)=\frac{4 \pi}{B_{M S}} \int_{-\infty}^{\infty} g\left(E^{\prime}\right) d E^{\prime} \int_{0}^{\infty} \frac{R^{2}}{\exp \left(2 \alpha R+\frac{\left|E-E_{F}\right|+\left|E^{\prime}-E_{F}\right|+\left|E-E^{\prime}\right|}{2 k_{B} T}\right)+\frac{1}{\sigma_{1}\left(E^{\prime}\right)}} d R .
$$

The denominator of the argument in the radial integral suppresses contributions due to hopping from sites with energies far from $E_{\mathrm{F}}$, and due to hopping to sites that are far away or for which $\sigma_{1}$ is small (hopping to sites at which carriers are effectively strongly trapped). So far, the MS model was used only for hopping between molecules with equal decay lengths of their wave functions, $\alpha^{-1}$. The tunnel rate between two sites varies then as $\exp (2 \alpha R)$ with the intersite distance $R$. The tunnel rate between two chemically different sites, with wave function decay lengths $\alpha^{-1}$ and $\beta^{-1}$, would be proportional to $\exp [(\alpha+\beta) R]$. In order to be able to model the mobility in a system with a bimodal DOS, $g(E)=g_{\mathrm{h}}(E)$ 


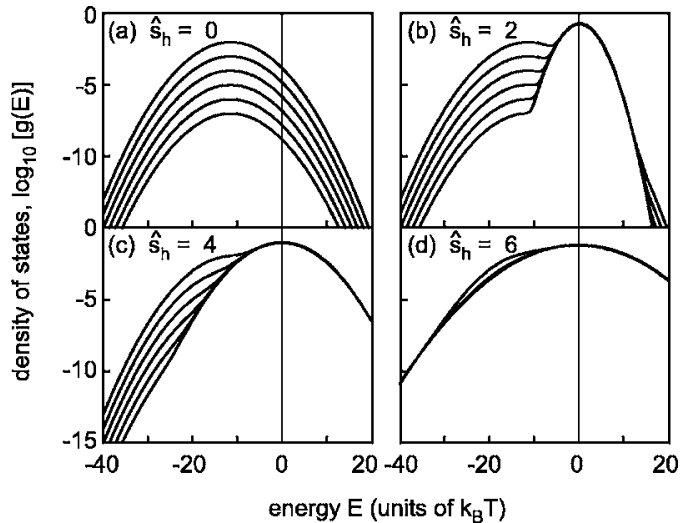

FIG. 2. Densities of states of host-guest systems with a bimodal Gaussian DOS, given on a $\log _{10}$ scale, for guest concentrations $x=10^{-6}, 10^{-5} \ldots 10^{-1}$. The energy difference $\Delta=11.51 k_{\mathrm{B}} T$ between the host and guest states is such that $x_{1 / 2}=\exp \left[-\Delta /\left(k_{\mathrm{B}} T\right)\right]=10^{-5}$. The reduced width of the guest DOS is $\hat{s}_{\mathrm{g}}=4$, and the reduced width of the host DOS is $\hat{s}_{\mathrm{h}}=0$ (a), $\hat{s}_{\mathrm{h}}=2$ (b), $\hat{s}_{\mathrm{h}}=4$ (c), and $\hat{s}_{\mathrm{h}}=6$ (d).

$+g_{\mathrm{g}}(E)$, with different wave function decay lengths for the host and guest molecules, $\alpha_{\mathrm{h}}^{-1}$ and $\alpha_{\mathrm{g}}{ }^{-1}$, respectively, we use Eq. (12) with an effective value of $2 \alpha$ that depends in the following way on the energies $E$ and $E^{\prime}$ :

$$
\begin{aligned}
2 \alpha_{e f f}\left(E, E^{\prime}\right)= & 2 \alpha_{h} \frac{g_{h}(E)}{g(E)} \frac{g_{h}\left(E^{\prime}\right)}{g\left(E^{\prime}\right)} \\
& +\left(\alpha_{h}+\alpha_{g}\right)\left(\frac{g_{h}(E)}{g(E)} \frac{g_{g}\left(E^{\prime}\right)}{g\left(E^{\prime}\right)}+\frac{g_{g}(E)}{g(E)} \frac{g_{h}\left(E^{\prime}\right)}{g\left(E^{\prime}\right)}\right) \\
& +2 \alpha_{g} \frac{g_{g}(E)}{g(E)} \frac{g_{g}\left(E^{\prime}\right)}{g\left(E^{\prime}\right)}
\end{aligned}
$$

This is a simple approximate method for taking the nature of the molecules that most strongly contribute to the hopping conduction into account, for any combination of initial and final state energies. Eq. (13) is exact in the zero host or guest concentration limits, and for the case of equal values of $\alpha_{\mathrm{h}}$ and $\alpha_{\mathrm{g}}$.

\section{RESULTS}

In order to demonstrate the relevance of the carrier concentration dependence of the mobility in host-guest systems, we first show the results of calculations of the mobility in a series of systems with a host DOS with a width that is varied from $\hat{s}_{\mathrm{h}}=0$ to $\hat{s}_{\mathrm{h}}=6$, and for a fixed width of the guest DOS, $\hat{s}_{\mathrm{g}}=4$. All calculations were done for the case $\exp \left[-\Delta /\left(k_{\mathrm{B}} T\right)\right]=10^{-5}$, as in Fig. 1, corresponding to $\Delta=0.29 \mathrm{eV}$ at room temperature. Figure 2 shows the corresponding total densities of states, on a $\log _{10}$ scale, for guest concentrations from $10^{-6}$ to $10^{-1}$. Figure 3 shows for these four systems contours of equal values of the $\log _{10}$ of the ratio $\mu(x, c) / \mu(0,0)$, calculated using the Martens model. The reference mobility is thus the mobility of the host material, in the limit of a very small carrier concentration ( $\left.c \ll c_{\text {crossover }}\right)$.

From a comparison between Figs. 1 and 3(a), it is seen that the carrier concentration range in which the mobility is

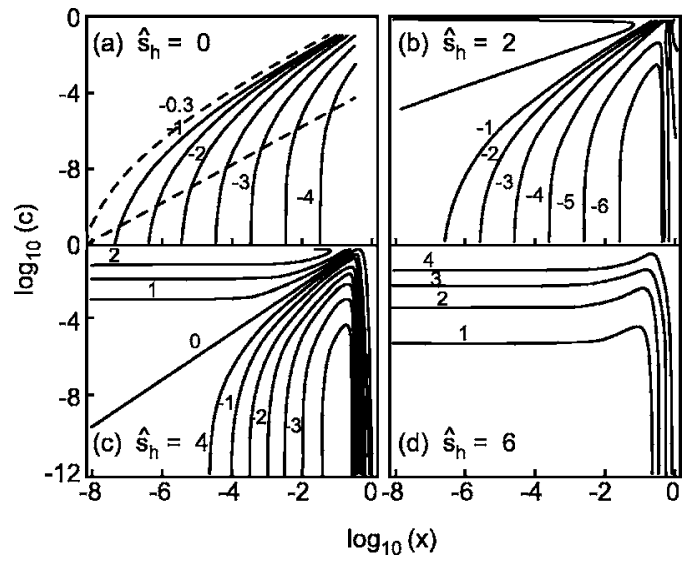

FIG. 3. Guest concentration $(x)$ and carrier concentration $(c)$ dependence of the mobility in a host-guest system with a bimodal Gaussian DOS with $\hat{s}_{\mathrm{g}}=4, x_{1 / 2}=\exp \left[-\Delta /\left(k_{\mathrm{B}} T\right)\right]=10^{-5}$, and $\hat{s}_{\mathrm{h}}=0$ (a), $\hat{s}_{\mathrm{h}}=2$ (b), $\hat{s}_{\mathrm{h}}=4$ (c), and $\hat{s}_{\mathrm{h}}=6$ (d). The figure shows contours of equal mobility, expressed as $\log _{10}[\mu(c, x) / \mu(0,0)]$, calculated from the Martens model. The shapes of the corresponding densities of states are given in Fig. 2.

strongly carrier concentration dependent (the region in between the dashed curves) is increased with the introduction of disorder of the guest molecules. The upper dashed curve is the $\mu(x, c) / \mu(0,0)=0.5$ contour line, and the lower dashed curve is the line at which the mobility is equal to two times the mobility in the $c=0$ limit. Disorder of the guest molecules also shifts the $x_{1 / 2}$ point, obtained in the small-c limit, to smaller values. The value $x_{1 / 2}=3.3 \times 10^{-9}$, predicted by Eq. (9), is found to agree excellently with the numerically calculated result.

When also the host DOS is disordered, the mobility does not only increase with $c$ upon filling of the guest states, but it also increases upon filling of the host states, viz. when $c>c_{\text {crossover }}$ (for $x=0$ ). This effect is visible in Figs. 3(b) and $3(\mathrm{c})$. With increasing width of the host DOS, the guest concentration range within which the mobility is reduced due to trapping at guest sites is reduced. For the $c=0$ limit, this is described by Eq. (9). Both figures also reveal the transition between the $\mathrm{HGH}$ and GG hopping regimes, at a guest concentration around $10^{-1}$, depending on the carrier concentration and on $\hat{s}_{\mathrm{h}}$.

When the host DOS becomes even wider, a point is reached at which the $(x, c)$ area within which the mobility is decreased (with respect to the reference mobility for small $x$ and $c$ ) vanishes. The overlap between the host and guest DOS is then very large, so that the guest DOS hardly affects the shape of the total DOS [see Fig. 2(d)]. For the set of parameters studied, this happens when $\hat{s}_{\mathrm{h}}=5.85$. Figure $3(\mathrm{~d})$ shows that for the case $\hat{s}_{\mathrm{h}}=6$ the mobility is for all $(x, c)$-points larger than the reference mobility.

Figure 4 shows more clearly than Fig. 3 how the mobility varies at large guest concentrations, around and above the crossover to the GG hopping regime. Figures 4(a) and 4(b) give results for the Boltzmann limit, and Fig. 4(c) shows the effect of the carrier concentration dependence of the mobility, by giving results for the Boltzmann limit (dashed curves) and for a carrier concentration $c=10^{-4}$. The thin full and 

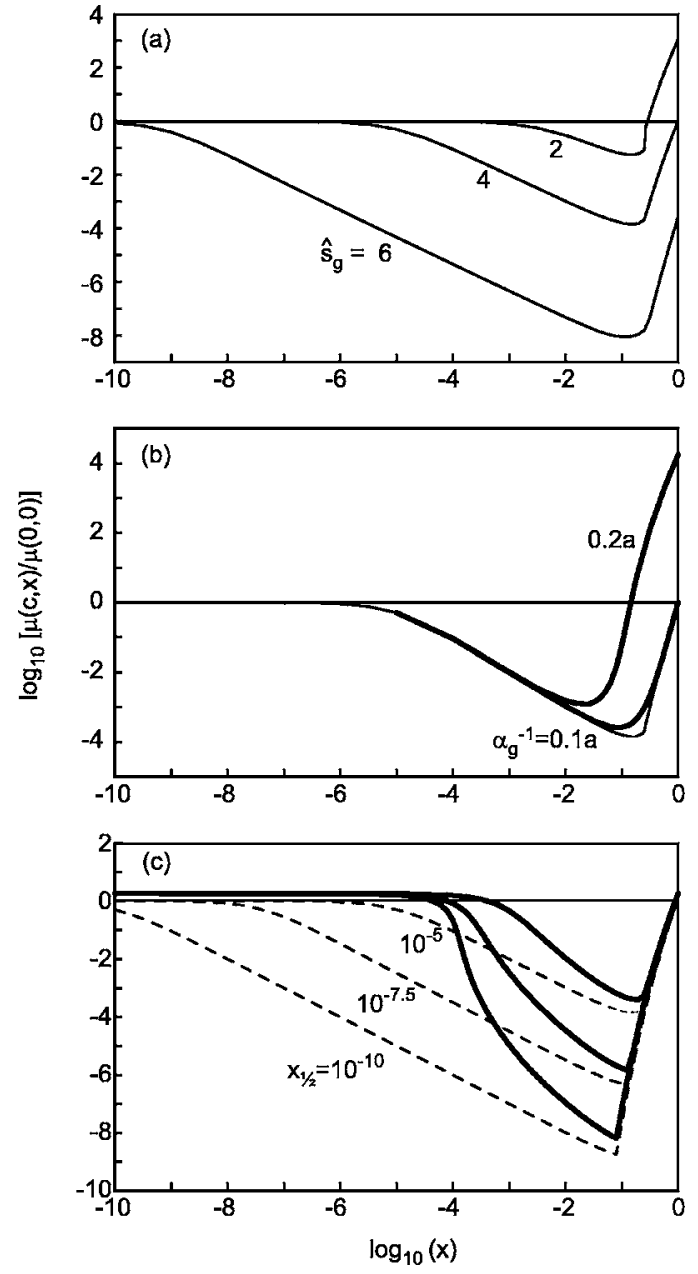

FIG. 4. The mobility in a bimodal Gaussian DOS, as a function of the guest concentration $(x)$ and as a function of various other parameter values, calculated from the Martens model (thin lines) and the Movaghar-Shirmacher model (thick lines). The default parameter values are $\hat{s}_{\mathrm{h}}=\hat{s}_{\mathrm{h}}=4, \quad \exp \left[-\Delta /\left(k_{\mathrm{B}} T\right)\right]=10^{-5}, \quad \alpha_{\mathrm{h}}^{-1}=\alpha_{\mathrm{g}}^{-1}$ $=0.1 a$, and $c \rightarrow 0$ (Boltzmann limit). (a) Dependence on the width of the guest DOS, $\hat{s}_{\mathrm{g}}$. (b) Dependence on the wave function decay length of the guest molecules, $\alpha_{g}^{-1}$. (c) Dependence on the displacement $\Delta$ (expressed as $x_{1 / 2}=\exp \left[-\Delta /\left(k_{\mathrm{B}} T\right)\right]$ ) between the host and guest densities of states, for the Boltzmann limit (dashed curves) and for $c=10^{-4}$ (full curves).

dashed curves were calculated using the Martens model, and the thick full curves were calculated using the MS model. The following set of default parameters was used: $\hat{s}_{\mathrm{h}}=\hat{s}_{\mathrm{g}}=4$, $\alpha_{\mathrm{h}}^{-1}=\alpha_{\mathrm{g}}{ }^{-1}=0.1 a$, and $\exp \left[-\Delta /\left(k_{\mathrm{B}} T\right)\right]=10^{-5}, \quad$ so that $\Delta /\left(k_{\mathrm{B}} T\right)=11.51$.

Figure 4(a) shows that a larger width of the guest DOS leads to a shift of the effective $x_{1 / 2}$ point to lower guest concentrations, as given by Eq. (9), and to a smaller mobility in the GG regime. These Martens model calculations reveal only a weak dependence of the guest concentration at which the mobility is at a minimum on the width of the guest DOS, and suggest that the HGH-GG transition can be quite abrupt. However, the lower two curves in Fig. 4(b) (for $\hat{s}_{\mathrm{g}}=4$ and $\alpha_{\mathrm{g}}^{-1}=0.1 a$ ) show that within the more sophisticated MS model the minimum is less deep, and shifted to a slightly

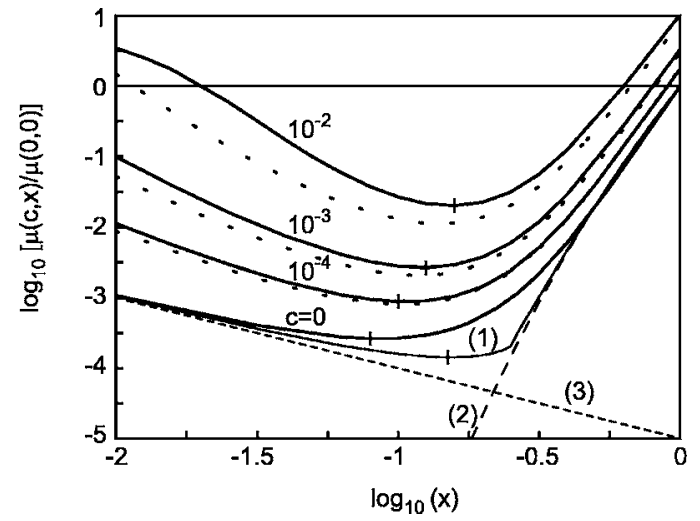

FIG. 5. The mobility in a bimodal Gaussian DOS, as a function of the guest concentration $(x)$ and for various values of the carrier concentration $(c)$, calculated from the Movaghar-Shirmacher model (thick lines). Parameter values: $\hat{s}_{\mathrm{h}}=\hat{s}_{\mathrm{h}}=4, \exp \left[-\Delta /\left(k_{\mathrm{B}} T\right)\right]=10^{-5}$, and $\alpha_{\mathrm{h}}^{-1}=\alpha_{\mathrm{g}}^{-1}=0.1 a$. The dotted lines have been obtained from the $c=0$ curve using Eq. (20). The thin line (1) is the result for $c=0$, obtained from the Martens model. The dashed lines (2) and (3) are approximations for small $c$ and large and small $x$, respectively, given by Eqs (18) and (16), respectively.

smaller guest concentration. The difference between the results from both models is only significant in a narrow concentration range around the minimum. The upper curve in Fig. 4(b) shows the mobility for the case of guest molecules for which the wave function extension is two times larger than that for the host molecules. As a result, the guest concentration at which the mobility is smallest is shifted from approximately $0.1(10 \%)$ to approximately $0.03(3 \%)$, and the mobility for the $x=1$ system is increased by more than four orders of magnitude.

The dashed curves in Fig. 4(c) (Boltzmann regime) show that in the HGH-regime an increase of $\Delta$ (so that $x_{1 / 2}$ $=\exp \left[-\Delta /\left(k_{\mathrm{B}} T\right)\right]$ is decreased) has a similar effect on the mobility curves as an increase of the width of the guest DOS [Fig. 4(a)]. When modeling experimental data, it is thus important to have independent information about at least one of these parameters. The full curves in Fig. 4(c) (for $c=10^{-4}$ ) show the large effect of the carrier concentration on the mobility curves:

(1) The guest concentration above which the mobility is reduced by a factor of two is strongly increased.

(2) In the HGH-regime the curves are not anymore always linear (on a double-log scale).

(3) In the HGH-regime, the average slope of the curves becomes more negative than -1 .

(4) Although the effect decreases with increasing guest concentration, a carrier concentration of $c=10^{-4}$ even increases the mobility around the mobility minimum $\left(x \sim 10^{-1}\right)$, viz. by a factor 3 to 4 . The position of the minimum is not strongly affected.

In Sec. V, some consequences for the analysis of experimental results are given.

In Fig. 5, the situation close to the mobility minimum is investigated in more detail. The thick curves show the carrier concentration dependence of the mobility for the case of an equal width of the host and guest $\operatorname{DOS}(\hat{s}=4)$, for equal wave 
function decay lengths $\left(\alpha^{-1}=0.1 a\right)$, and for $\exp \left[-\Delta /\left(k_{\mathrm{B}} T\right)\right]$ $=10^{-5}$ [as for the two lower curves in Fig. 4(b)], calculated using the MS model. It is seen that the carrier concentration dependence of the mobility increases with decreasing guest concentration. This is explained in the next section. As a consequence, $x_{\min }$ increases with increasing carrier concentration, from $\sim 0.09$ in the Boltzmann limit to $\sim 0.16$ for $c=10^{-2}$ for the set of parameters used. The thinner curve (1) shows the result from the Martens model for the mobility in the Boltzmann limit. The predictions from both models are only significantly different in the range $0.05<x<0.5$, in which the crossover to the direct guest-to-guest hopping regime takes place.

\section{ANALYSIS}

In this section a more quantitative analysis is given of the results shown in Sec. III.

Figure 3 revealed that there is a critical value for the width of the host DOS, beyond which the presence of guest molecules with a certain fixed width and energetic shift of the DOS does not give rise to a decrease of the mobility. An estimate for this critical value can be obtained by taking in Eq. (9) $x_{1 / 2}$ equal to $x_{\min }$, the guest concentration at which the mobility is minimal. This suggests the criterion

$$
\hat{s}_{h}>\sqrt{2 \frac{\Delta}{k_{B} T}+\hat{s}_{g}^{2}+2 \ln x_{\min }} .
$$

Taking $x_{\min } \approx 0.1$ [from Fig. 3(c)], the critical value of $\hat{s}_{\mathrm{h}}$ would then be equal to 5.87 , for the parameter set used in Fig. 3. The agreement with the exactly calculated value (5.85) is excellent. Eq. (14) was also found to provide a good description of the variation of the crossover width of the host DOS for smaller values of $\hat{s}_{\mathrm{g}}$, in the range from 0 to 4 .

Figures 3(b) and 3(c) show that contour lines for which $\mu(x, c)=\mu(0,0)$ (so that $\log _{10}[\mu(x, c) / \mu(0,0)]=0$ ) are to a good approximation straight lines over a large part of the diagrams. These lines connect $(x, c)$-points at which the decrease of the mobility by the introduction of guest molecules is precisely compensated by the increase of the mobility due to the carrier concentration dependence of the mobility. We have not been able to derive general expressions for these contour lines. The empirical expression

$$
\log _{10} c=\left[\frac{1}{2}+\frac{1}{2}\left(\frac{\sigma_{D O S}}{k_{B} T}\right)^{2} /\left(\frac{\Delta}{k_{B} T}\right)\right] \log _{10} x,
$$

provides a good description of the zero contours for systems with $\hat{s}_{\mathrm{h}}=\hat{s}_{\mathrm{g}}$, and with parameter values close to those used in Fig. 3(c). For $\hat{s}=4$ and $\exp \left[-\Delta /\left(k_{\mathrm{B}} T\right)\right]=10^{-5}$, the slope of the zero contour that follows from Eq. (15) is equal to 1.195, in excellent agreement with the numerically calculated value.

Figure 4 and line (1) in Fig. 5 show that the guestconcentration dependent mobility curves as calculated from the Martens model can show a pronounced change of the slope at the crossover between the two transport regimes. We find that such discontinuities are accompanied by a simultaneous change of the energy $E^{*}$ and the distance $R^{*}$ (see Sec. II). E.g., line (1) in Fig. 5 shows a discontinuous slope at $x$

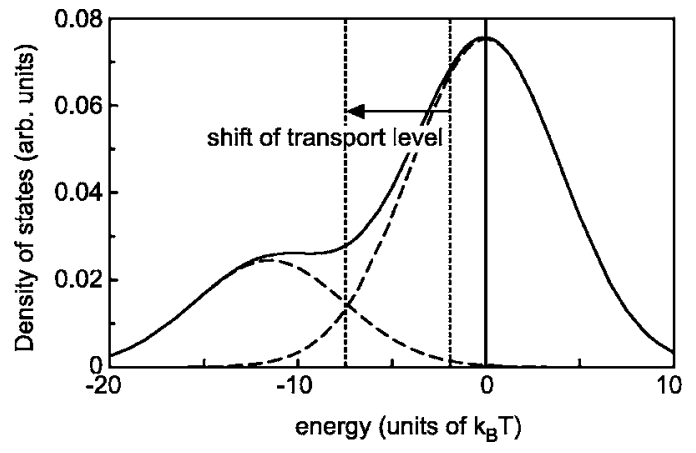

FIG. 6. Shift of the transport level, as calculated from the Martens model, at the crossover guest concentration $x=0.24$ for line (1) in Fig. 5, for the case $\hat{s}_{\mathrm{h}}=\hat{s}_{\mathrm{h}}=4, \exp \left[-\Delta /\left(k_{\mathrm{B}} T\right)\right]=10^{-5}, \alpha_{\mathrm{h}}^{-1}=\alpha_{\mathrm{g}}^{-1}$ $=0.1 a$, and a carrier concentration well below the crossover value (Boltzmann limit). The figure also shows the total density of states (full line) and the partial host and guest densities of states (dashed lines).

$=0.24$. At that point the transport energy decreases with increasing $x$ from approximately $-1.9 \times k_{\mathrm{B}} T$ to $-7.6 \times k_{\mathrm{B}} T$. This shift is depicted in Fig. 6, which also shows the DOS at this guest concentration. The transport level thus shifts from a position well in the host DOS (guest-limited hopping regime) to an energy where the guest DOS is slightly larger than the host DOS (guest-to-guest hopping regime). At the same time, $R^{*}$ increases from $\sim 1.00 \times a$ to $\sim 1.25 \times a$. We regard the results from the MS model as more accurate than the Martens model, because the function $\sigma_{1}(E)$ that is used within that model (see Sec. II B) gives states at any energy a certain (energy-dependent) weight, whereas the mobility as calculated from the Martens model depends only on the density of states below a certain energy $\left(E^{*}\right)$. Within the MS model, the transition is therefore somewhat smeared out. As the mobility curve is strongly asymmetric in the region close to the minimum, the minimum is shifted downward, from approximately $x_{\min }=0.15$ (Martens model) to $x_{\min }=0.09$ (MS model).

As a rough, first quantitative estimate of the value of $x_{\min }$, one might take the guest concentration at which the curves that describe the mobility deep in the HGH and GG hopping regimes cross. We first discuss this approach for the case of very small carrier concentrations (Boltzmann regime). The mobility in the HGH-regime is given by Eq. (9). For sufficiently large values of $\Delta$ and/or $\sigma_{\mathrm{DOS}, \mathrm{g}}$ (as compared to $\left.\sigma_{\mathrm{DOS}, \mathrm{h}}\right)$, so that the guests effectively act as deep traps, Eq. (9) can be simplified to

$$
\mu_{g c}(x, 0) \cong \frac{\exp \left[-\frac{\Delta}{k_{B} T}-\frac{1}{2} \hat{s}_{g}^{2}+\frac{1}{2} \hat{s}_{h}^{2}\right]}{x} \mu(0,0) .
$$

Using Eq. (23) in Ref. 19, the $x$ dependence of the mobility in the guest-to-guest hopping regime may for small $\hat{S}_{\mathrm{g}}$ be written as

$$
\mu_{g g}(x, 0) \cong C_{g} \exp \left[-\frac{1}{x^{1 / 3}}\left(\frac{6 B}{\pi} \frac{\alpha_{g}}{N_{t}}\right)^{1 / 3}\right] .
$$

where the prefactor $C_{\mathrm{g}}$ depends on $\sigma_{\mathrm{DOS}, \mathrm{g}}, T, \nu_{0}$, and $N_{\mathrm{t}}$. The reference mobility, $\mu(0,0)$, is given by an expression analo- 
gous to Eq. (17), with $x=1$. Assuming, for simplicity, that $C_{\mathrm{h}}=C_{\mathrm{g}}$, it follows that in the guest-to-guest hopping regime

$$
\mu_{g g}(x, 0) \cong \exp \left[-\left(\frac{6 B}{\pi} \frac{\alpha_{g}}{N_{t}}\right)^{1 / 3}\left(\frac{1}{x^{1 / 3}}-\frac{\alpha_{h}^{1 / 3}}{\alpha_{g}^{1 / 3}}\right)\right] \mu(0,0)
$$

This analysis is applied in Fig. 5, where curve (2) gives the mobility as predicted by Eq. (18), with $B=1.80$ as the parameter that best fits the large- $x$ part of the result from the Martens model [curve (1)], and curve (3) gives the mobility for small $x$ [Eq. (16)]. Curve (2) provides a good approximation for large concentrations. The intersection between curves (2) and (3) occurs for a guest concentration that is close to the value where the mobility from the Martens model shows a clear change of the slope. Qualitatively, also the trends in $x_{\text {min }}$ that are observed in Fig. 4 when varying $\hat{s}_{\mathrm{g}}, \alpha_{\mathrm{g}}^{-1}$, and $\Delta$ are explained well from the intersection method, described above. However, the more precise values, obtained from the MS method, tend to be systematically somewhat smaller.

The observed upward shift of $x_{\min }$ with increasing carrier concentration (Fig. 5) can be explained from the following simple model. The carrier concentration dependence of the mobility at a fixed value of $x$ can, to a first approximation, be obtained using the approximation that the transport level is fixed (see Sec. II). The mobility enhancement is then only determined by the variation of the Fermi level energy with $c$ [see Eq. (6)]. When the host DOS at $E_{\mathrm{F}}$ is small with respect to the guest DOS (i.e., when the host DOS is sufficiently narrow, the displacement between the host and guest DOS is sufficiently large, and the carrier density is sufficiently small), $E_{\mathrm{F}}(x, c)$ is only determined by the shape of the guest DOS. The mobility enhancement is then given by an expression that is analogous to that given by Eq. (28) in Ref. 19 for a unimodal Gaussian DOS

$$
\frac{\mu(x, c)}{\mu(x, 0)} \cong \frac{x}{c} \exp \left[\frac{E_{F, g}\left(\frac{c}{x}\right)}{k_{B} T}+\frac{1}{2} \hat{s}_{g}^{2}\right],
$$

where $E_{\mathrm{F}, \mathrm{g}}(c / x)$ is the Fermi energy in the normalized guest DOS, centered at $E=0$, for a carrier concentration $c / x$. Equation (19) expresses that, under the conditions considered, the mobility enhancement is governed by the same expression as for the case of a unimodal DOS, provided that the carrier concentration is renormalized by a factor $1 / x$. This explains why the enhancement increases with decreasing $x$, and why $x_{\text {min }}$ increases with increasing carrier concentration, as noted already above. The carrier concentration at which the mobility is enhanced by a factor 2 is given by the renormalized version of Eq. (2), i.e., $c_{\text {crossover }}(x)=(x / 2) \exp \left(-\hat{s}_{\mathrm{g}}^{2} / 2\right)$. In Fig. $3(\mathrm{a})$, these crossover concentrations are indicated by the lower dashed line.

In Ref. 19, a simple analytical expression was given that provides a good approximation to the right-hand-side part of Eq. (19). For the case of a host-guest system with $\hat{s}_{\mathrm{h}}=\hat{s}_{\mathrm{g}}=\hat{s}$, it would have the form

$$
\frac{\mu(x, c)}{\mu(x, 0)} \cong \exp \left[\frac{1}{2}\left(\hat{s}^{2}-\hat{s}\right)\left(\frac{2 c}{x}\right)^{\delta}\right]
$$

with

$$
\delta \cong 2 \frac{\ln \left(\hat{s}^{2}-\hat{s}\right)-\ln [\ln (4)]}{\hat{s}^{2}} .
$$

Using this approach, and the MS result for $c=0$, the dotted curves shown in Fig. 5 are obtained. The approach is very good for values of $c / x$ up to almost $10^{-2}$, i.e., for the host material up to $c=10^{-2}$ and near the mobility minimum up to $c=10^{-3}$. It can also be concluded from the figure that the upward shift of $x_{\min }$ with the carrier concentration is quite accurately predicted by the model.

\section{EFFECT OF THE CARRIER CONCENTRATION ON THE TIME-OF-FLIGHT MOBILITY}

Extensive systematic studies of the guest concentration dependence of the mobility in organic host-guest systems have been carried out in the 1990s by the Borsenberger group at Kodak, ${ }^{6}$ using time-of-flight (TOF) experiments. This has resulted in important novel insights, e.g., concerning the origin of energetic disorder (see e.g., Ref. 25) and concerning the effect of disorder on the hopping mobility. ${ }^{10}$ However, for relatively large trap depths, above $0.25 \mathrm{eV}$, important discrepancies between the experimental and theoretical $\mu(x)$ curves were found to remain. ${ }^{15,16}$ This can be seen from Fig. 6, which gives the TOF mobilities for three prototype systems, reproduced from Borsenberger et al., ${ }^{16}$ consisting of an inert (nonconductive) polystyrene matrix, di$p$-anisyl- $p$-tolylamine (DAT), di- $p$-tolylphenylamine (DTP) or diphenyl- $p$-tolylamine (DPT), which act as a host material, and tri-anisylamine (TAA), which acts as the guest material.

TAA molecules are hole traps in all systems studied, with $\Delta=0.07, \quad 0.27$, and $0.34 \mathrm{eV}$ as obtained from cyclic voltammetry $^{16}$ in the DAT, DTP, and DPT hosts, respectively. Borsenberger et al. expressed their surprise about two observations for the TAA:DPT system:

(1) The slope of the $\log _{10}(\mu)$ versus $\log _{10}(x)$ curve is approximately -1.5 , whereas from the generalized HL formula [Eq. (8)] a value of -1 is expected, and

(2) The effective $x_{1 / 2}$ concentration is for the TAA:DPT system almost two orders of magnitude larger than the value $x_{1 / 2}=\exp \left[-\Delta /\left(k_{\mathrm{B}} T\right)\right]=1.2 \times 10^{-6}$ that would be expected from Eq. (8), if the width of the host and guest DOS would be equal.

It follows from Eq. (8) that the observed value of $x_{1 / 2}$ could, in principle, be explained as the result of a different Gaussian width of the host and guest DOS. However, that would not explain the large negative slope of the mobility curve.

We show in this section that these observations can be understood as a result of a carrier concentration dependence of the mobility. In a typical TOF experiment, such as described in Ref. 16, a thin sheet or charge carriers is created by a short optical excitation in a $\sim 100 \mathrm{~nm} \alpha$-Se holegeneration layer near one of the electrodes, in a stacked 
electrode/semiconductor/electrode device over which a voltage difference $V$ is applied. The optimum between a large signal but small space charge effects is typically obtained for areal carrier densities around $n_{\mathrm{a}}=2 \times 10^{-2} C_{\mathrm{a}} V / q$, where $C_{\mathrm{a}}$ $=\varepsilon / L$ is the capacitance per unit area. Here, $\varepsilon$ is the dielectric constant (typically, $\varepsilon_{\mathrm{r}}=3$ ) and $L$ the device thickness (typical value: $L=10 \mu \mathrm{m} ; V$ is then typically $300 \mathrm{~V}$ ). It follows that $n_{\mathrm{a}} \approx 10^{14} \mathrm{~m}^{-2}$. The initial width of the charge sheet is determined by the optical absorption and carrier mobility in the hole-generation layer. It may be assumed to be around $100 \mathrm{~nm}$, or less. When the charge sheet moves to the other electrode, its shape changes. In the case of a constant mobility and diffusion coefficient, diffusion leads to a symmetric broadening, and to a decrease of the peak concentration. Assuming that the Einstein relation is valid, and that the initial sheet would have a $\delta$-function shape, the diffusive broadening would lead to a width $\Delta x=\sqrt{\left[k_{\mathrm{B}} T /(q V)\right] \times L}$ at the exit electrode. For $V=300 \mathrm{~V}$ and $T=300 \mathrm{~K}$, the width is then $1.0 \%$ of the device thickness, so for $L=10 \mu \mathrm{m}$, the width would be $100 \mathrm{~nm}$. If the initial width of the charge sheet is indeed around $100 \mathrm{~nm}$, or less, its average width during transfer through the layer is roughly $\sim 100-200 \mathrm{~nm}$. The average carrier density is then $\sim 10^{21} \mathrm{~m}^{-3}$. For the host-guest systems considered in Fig. 6, the site density was kept fixed at $7.2 \times 10^{26} \mathrm{~m}^{-3} \cdot{ }^{16}$ It is therefore estimated that the average carrier concentration is $\sim 10^{-6}$. If the mobility and diffusion constant are carrier concentration dependent, the broadening is not symmetric. The development of a theory for that effect is beyond the scope of this paper.

Figure 7 (full curves) shows that for all hosts, the guest concentration dependence of the mobility can be well understood from our mobility model, using $c=10^{-6}$, as estimated above, using values of $\Delta$ that are within the experimental uncertainty equal to the experimental values (see the figure caption), and assuming that $\hat{s}_{\mathrm{h}}=\hat{s}_{\mathrm{g}}=5$. The Gaussian widths assumed are quite realistic for the systems considered. ${ }^{25} \mathrm{We}$ have not made an attempt to refine the fits by considering the possibility that the host and guest DOS have a different width. The dashed curves give the result for the zeroconcentration limit. The carrier concentration dependence is most important for the systems with a DPT host, for which the effect shifts the effective value of $x_{1 / 2}$ by two orders of magnitude. The agreement between the model results and the experimental results is excellent. Therefore, we regard this as strong support for our point of view that taking the carrier concentration dependence of the mobility into account can be important when interpreting the observed TOF-mobilities in host-guest systems.

\section{SUMMARY AND CONCLUSIONS}

In this paper, we have shown that the mobility of charge carriers in disordered organic host-guest systems depends on the carrier concentration. The effect has been studied on the basis of two theoretical models. The relatively simple Motttype model developed by Martens et al. provides an excellent description of the effect in the separate $\mathrm{HH}, \mathrm{HGH}$, and GG hopping regimes. The much more sophisticated, but computationally less efficient Movaghar-Shirmacher method

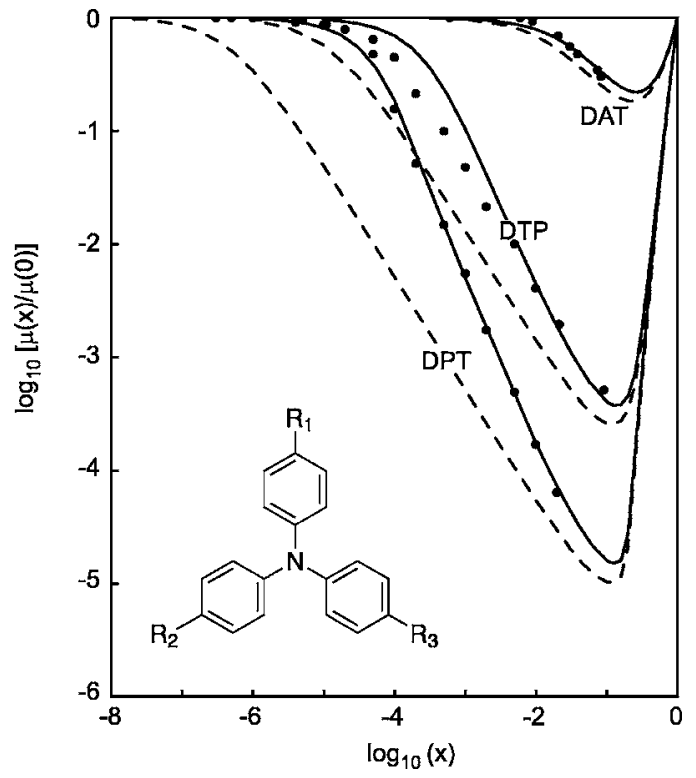

FIG. 7. Comparison of calculated (Martens model) and measured (Ref. 16) hole mobilities for systems consisting of a polystyrene matrix, DAT, DTP, and DPT host molecules, and TAA guest molecules. The chemical structures are as shown in the inset, with $R_{1}=R_{2}=R_{3}=\mathrm{OCH}_{3}$ for TAA, $R_{1}=\mathrm{CH}_{3}$ and $R_{2}=R_{3}=\mathrm{H}_{3}$ for DAT, $R_{1}=R_{2}=\mathrm{CH}_{3}$, and $R_{3}=\mathrm{H}$ for DTP, and $R_{1}=\mathrm{CH}_{3}$ and $R_{2}=R_{3}=\mathrm{H}$ for DPT. Full lines: $\hat{s}_{\mathrm{h}}=\hat{s}_{\mathrm{h}}=5, \alpha_{\mathrm{h}}^{-1}=\alpha_{\mathrm{g}}^{-1}=0.1 a, \Delta=0.10 \mathrm{eV}$ (DAT), $0.28 \mathrm{eV}$ (DTP), $0.36 \mathrm{eV}$ (DPT), $T=300 \mathrm{~K}$, for $c=10^{-6}$. Dashed lines: the same parameters, for $c=0$.

provides a better prediction for guest concentrations close to the mobility minimum, around which the cross-over between the HGH and GG hopping regimes takes place. Using the Movaghar-Shirmacher method, it is also possible to model cases in which the wave function extensions of the host and guest molecules are different. By a detailed analysis of measured data for hole-transporting hole-guest systems, taken from the literature, it has been made plausible that this effect can have a large influence on the TOF-mobility, measured under realistic experimental conditions. Host-guest systems are employed intensively in OLEDs, where fluorescent or phosphorescent dyes are embedded in an organic matrix material with a larger HOMO-LUMO gap. Typical dye concentrations vary from less than $0.1 \%$ to approximately $10 \%$. In OLEDs that are based on a multilayer stack, containing blocking layers that confine the emission zone to a thin central layer, the carrier concentrations in that layer can be as high as $10^{-3}$. This work suggests that only device models that take the large carrier concentration dependence of the mobility that can occur under such conditions into account, could yield a realistic description of the device performance. We remark that the theoretical framework developed in this paper can be extended straightforwardly to more complex host-guest systems, containing more than one dye. A first limitation of the present study is that it is based on the assumption that all carriers are in quasi-thermodynamic equilibrium. For systems with very deep traps, leading to release times that exceed typical experimental measurement times, the models used are not adequate. A second limitation is that 
only the mobility in the limit of zero applied field has been considered. It will be of interest to study that effect using the master equation method, applied successfully by Pasveer et al. to the case of a unimodal Gaussian DOS. ${ }^{20}$

\section{ACKNOWLEDGMENTS}

The author thanks P. A. Bobbert, W. F. Pasveer, and S. L. M. van Mensfoort for useful discussions.
${ }^{1}$ S. Doi, T. Yamada, Y. Tsubata, and M. Ueda, Proc. SPIE 5519, 161 (2004).

${ }^{2}$ R. Coehoorn, S. Vulto, S. L. M. van Mensfoort, J. Billen, M. Bartyzel, H. Greiner, and R. Assent, Proc. SPIE 6192, 619200 (2006).

${ }^{3}$ G. Cerullo, N. Nisoli, S. Stagira, S. de Silvestri, G. Lanzani, W. Graupner, E. List, and G. Leising, Chem. Phys. Lett. 288, 516 (1998), and references therein.

${ }^{4}$ M. A. Baldo, D. F. O'Brien, Y. You, A. Shousikov, S. Sibley, M. E. Thompson, and S. R. Forrest, Nature (London) 395, 151 (1998).

${ }^{5}$ M. Berggren, A. Dodabalapur, R. E. Slusher, and Z. Bao, Nature (London) 389, 466 (1997).

${ }^{6}$ P. M. Borsenberger and D. S. Weiss, Organic Photoreceptors for Xerography (Marcel Dekker, New York, 1998).

${ }^{7}$ D. M. Pai, J. F. Yanus, and M. Stolka, J. Phys. Chem. 88, 4717 (1984).

${ }^{8}$ D. C. Hoesterey and G. M. Letson, J. Phys. Chem. Solids 24, 1609 (1963).

${ }^{9}$ P. M. Borsenberger, E. H. Magin, and S. A. Visser, Jpn. J. Appl. Phys., Part 1 37, 1945 (1998).

${ }^{10}$ U. Wolf, H. Bässler, P. M. Borsenberger, and W. T. Gruenbaum, Chem. Phys. 222, 259 (1997).

${ }^{11}$ P. M. Borsenberger, W. T. Gruenbaum, U. Wolf, and H. Bässler, Chem. Phys. 234, 277 (1998).

${ }^{12}$ I. I. Fishchuk, A. K. Kadashchuk, H. Bässler, and D. S. Weiss, Phys. Rev. B 66, 205208 (2002).

${ }^{13}$ V. I. Arkhipov, P. Heremans, E. V. Emelianova, G. J. Adriaenssens, and H. Bässler, J. Phys.: Condens. Matter 14, 9899 (2002).
${ }^{14}$ I. I. Fishchuk, A. K. Kadashchuk, A. Vakhnin, Yu. Korosko, H. Bässler, B. Souharce, and U. Scherf, Phys. Rev. B 73, 115210 (2006).

${ }^{15}$ P. M. Borsenberger, W. T. Gruenbaum, E. H. Magin, S. A. Visser, and D. E. Schildkraut, J. Polym. Sci., Part B: Polym. Phys. 37, 349 (1999).

${ }^{16}$ P. M. Borsenberger, W. T. Gruenbaum, E. H. Magin, D. E. Schildkraut, and S. A. Visser, Jpn. J. Appl. Phys., Part 1 38, 117 (1999).

${ }^{17}$ M. C. J. M. Vissenberg and M. Matters, Phys. Rev. B 57, 12964 (1998).

${ }^{18}$ C. Tanase, E. J. Meijer, P. W. M. Blom, and D. M. de Leeuw, Phys. Rev. Lett. 91, 216601 (2003); C. Tanase, P. W. M. Blom, and D. M. de Leeuw, Phys. Rev. B 70, 193202 (2004).

${ }^{19}$ R. Coehoorn, W. F. Pasveer, P. A. Bobbert, and M. A. J. Michels, Phys. Rev. B 72, 155206 (2005).

${ }^{20}$ W. F. Pasveer, J. Cottaar, C. Tanase, R. Coehoorn, P. A. Bobbert, P. W. M. Blom, D. M. de Leeuw, and M. A. J. Michels, Phys. Rev. Lett. 94, 206601 (2005).

${ }^{21}$ R. Coehoorn (unpublished).

${ }^{22}$ H. C. F. Martens, I. N. Hulea, I. Romijn, H. B. Brom, W. F. Pasveer, and M. A. J. Michels, Phys. Rev. B 67, 121203(R) (2003).

${ }^{23}$ B. Movaghar and W. Schirmacher, J. Phys. C 14, 859 (1981).

${ }^{24}$ V. I. Arkhipov, I. I. Fishchuk, A. Kadashchuk, and H. Bässler, in Photophysics of Molecular Materials, edited by G. Lanzani (Wiley-VCH, Weinheim, 2005), Ch. 6, pp. 261-366.

${ }^{25}$ S. A. Visser, W. T. Gruenbaum, E. H. Magin, and P. M. Borsenberger, Chem. Phys. 240, 197 (1999). 